\title{
Extended Model-Based Automatic Gait Recognition of Walking and Running
}

\author{
Chew-Yean Yam, Mark S. Nixon, John N. Carter \\ Department of Electronics and Computer Science, \\ University of Southampton, S017 1BJ Southampton, United Kingdom. \\ \{cyy99r|msn|jnc\} decs.soton.ac.uk
}

\begin{abstract}
Gait is an emerging biometric. Current systems are either holistic or feature based and have been demonstrated to be able to recognise people by the way they walk. This paper describes a new system that extends the feature based approach to recognise people by the way they walk and run. A bilateral symmetric and coupled oscillator is the key concept that underlies this model, which includes both the upper and the lower leg. The gait signature is created from the phase-weighted magnitude of the lower order Fourier components of both the thigh and knee rotation. This technique has proved to be capable of recognising people when walking or running and future work intends to develop invariance attributes of walking or running for the new description.
\end{abstract}

\section{Introduction}

Using gait as a biometric is motivated by occlusion of criminals' faces and that they either walk or run to escape a crime scene. As such, even though many techniques have been developed to recognise people by the way they walk, there is no extant technique, which could recognise both by walking and by running. We describe a new system that can model both running and walking.

In literature, Aristotle and Leonardo da Vinci studied human movement and Shakespeare observed the possibility of recognition by gait. More recently, Murray ${ }^{[1]}$ produced standard walking movement patterns for pathologically normal men. Bianchi $^{[2]}$ revealed that there are inter-individual differences in human gait mechanics. Since the action of walking is dictated by the skeleto-muscular structure and the same structure is applied to running, if it suggests that if gait is indeed unique, then so should running. Perhaps, the earliest approach to gait recognition was to derive a gait signature from a spatio-temporal pattern ${ }^{[3]}$. Images were projected into an eigenspace and the resulting eigenvectors were used for recognition ${ }^{[4]}$. Then, the dense optical flow ${ }^{[5]}$ technique used the relative phases of optical flow to form a feature vector to create a signature. A more recent statistical based approach combined canonical space transformation based on canonical analysis with the eigenspace transformation. Later, temporal information obtained from optical-flow changes between two consecutive spatial templates was incorporated to improve recognition capability ${ }^{[6]}$. The only model-based human gait recognition system ${ }^{[7]}$ 
models human walking as two interconnected pendula representing thigh motion, which combined a velocity Hough transform with a Fourier representation to obtain a gait signature.

Walking may be described in terms of double support, where two limbs are in contact with the ground, and single support, where one foot is in contact with the ground. Running is the natural extension of walking, which involves increased velocities, different joint movement and coordination. The running cycle, however, is not distinguished from walking by velocity, but by whether a person becomes airborne during motion, with 2 periods of double float where neither foot is in contact with the ground. The way the foot contacts the ground is different for walking and for running. $\mathrm{Li}$ et. al. observed that there occur topological similarities in the coordination patterns between the thigh and lower leg in walking and running, which co-existed with functional differences throughout the gait cycle, especially in the transition from stance to swing phase., i.e. between $20 \%$ and $40 \%$ of the gait cycle ${ }^{[8]}$.

We describe a new gait model that can handle running and walking, and with fewer parameters, in section 2.1. We then show how this can be used to create a gait signature in section 2.2 , that is shown to be able to recognise people by the way they walk and run in section 3 , on a limited database.

\section{Gait Modelling and Analysis}

\subsection{Coupled Oscillator Gait Model}

As human gait is rhythmic and is naturally an oscillatory behaviour, ${ }^{[9]}$ we can assume that an oscillator controls each limb and that limb movement is interconnected or coupled in some way. The main characteristic of human gaits, including walking, running and sprinting is bilaterally symmetric where the left and right legs and opposite side of arms interchange with each other with a phase shift of half a period. Both legs perform the same motion but out of phase with each other by half a period. These motions operate in space and time, satisfying the rules of spatial symmetry (swapping legs) and temporal symmetry (a phase-lock of half a period in general). Fig. 1 shows the rotation both of thighs and knees for a walking subject. Hence, we can assume the legs are coupled oscillators with half a period of phase shift. Both legs can be modelled by two distinct but systematically coupled oscillators, which oscillate at the same frequency (frequency-lock) but with fixed relative phase difference.

The leg can be modelled as two pendula joined in series, see Fig. 2. The thigh rotation, $\theta_{T}(t)$, is described by Eq. (1), where $t$ is the time index for the normalised gait cycle, $A_{T}$ is the amplitude of the thigh rotation and $C_{T}$ is the offset. 

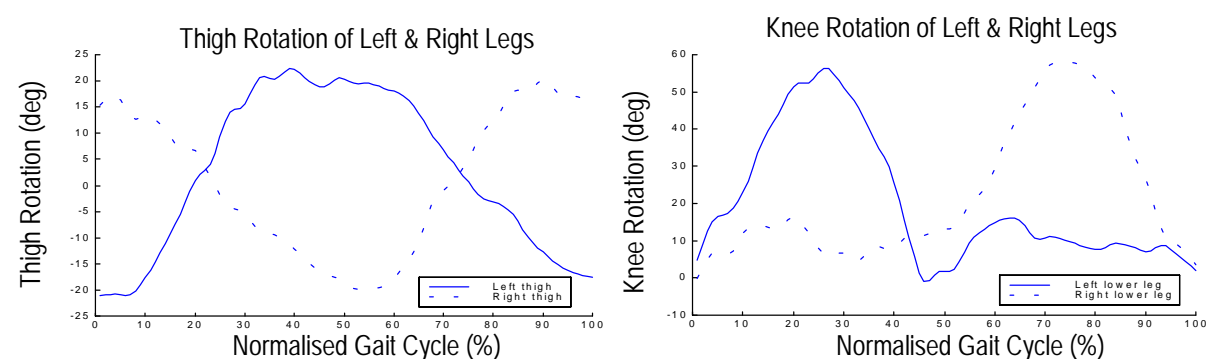

Fig. 1. (a) and (b) are the rotation of thighs and knees respectively, with half a period shift.

h

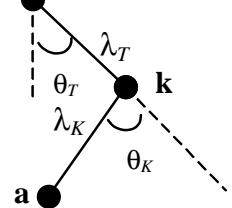

Fig. 2. The model of the thigh and lower leg: an upper pendulum models the thigh and the lower pendulum models the lower leg, connected at the knee joint.

$$
\theta_{T}(t)=A_{T} \cos (2 \pi t)+C_{T}
$$

Eq. (1) can be applied for both running and walking. Note that the gait cycles for running and walking are normalised so that they are invariant to speed. The knee rotation, $\theta_{K}(t)$, can be represented as

$$
\theta_{K}(t)=\left\{\begin{array}{cc}
A_{K 1} \sin ^{2}(2 \pi t)+C_{K 1} \quad, 0 \leq t<p \\
A_{K 2} \sin ^{2}(2 \pi(t+\phi))+C_{K 2}, p \leq t<1
\end{array}\right.
$$

where $A_{K 1}$ and $A_{K 2}$ are the amplitudes of the knee rotation, $C_{K 1}$ and $C_{K 2}$ are the offsets, $\phi$ is the phase shift and $p$ is the time when the second double support starts (walking) and the double float starts (running). For walking, $p$ is 0.4 whereas the thigh swings faster in running and then $p$ is 0.3 . Future work will aim to determine the effect of velocity on $p$. The $\sin ^{2}$ term models well the basic motion as depicted in Fig. 1(b).

Given subject extraction, where the horizontal position is known or fixed, the horizontal displacement of the hip can be ignored. However, the vertical oscillation made during running is bigger than for walking, thus, the vertical displacement, $S_{y}(t)$, needs to be incorporated in the model, as given by

$$
S_{y}(t)=A_{y} \sin (4 \pi t)
$$

where $A_{y}$ is the amplitude of the vertical oscillation. Note that the frequency is twice the frequency of the leg motion, as a gait cycle comprises of two steps.

The structure of the thigh can be described by a point $\mathbf{h}$ that represents the hip and the line passing through $\mathbf{h}$ at an angle $\theta_{T}$. The knee is then

$$
\mathbf{k}(t)=\mathbf{h}(t)+\lambda_{T} \mathbf{u}_{T}(t)
$$

where $\mathbf{u}_{T}(t)$ is the unit vector of the line direction, $\mathbf{h}$ is the position of the hip and $\lambda_{T}$ is the thigh length, as $\mathbf{u}_{T}(t)=\left[-\sin \theta_{T}(t), \cos \theta_{T}(t)\right]$ and $\mathbf{h}(t)=\left[h_{x}(0), h_{y}(0)+S_{y}(t)\right]$, where 
$h_{x}(0)$ and $h_{y}(0)$ are the initial hip coordinates. Decomposing Eq. (4) into the $x$ and $y$ parts yields the coordinates of the knee point as,

$$
\begin{gathered}
k_{x}(t)=h_{x}(0)-\lambda_{T} \sin \theta_{T}(t) \\
k_{y}(t)=h_{y}(0)+S_{y}(t)+\lambda_{T} \cos \theta_{T}(t)
\end{gathered}
$$

Similarly, the structure of the lower leg is given by a line which starts at the knee, that passes through $\mathbf{k}$ at an angle $\theta_{k}$. The ankle $\mathbf{a}$ is

$$
\mathbf{a}(t)=\mathbf{k}(t)+\lambda_{K} \mathbf{u}_{K}(t)
$$

where $\mathbf{u}_{K}(t)$ is the unit vector of the line direction, $\mathbf{k}(t)$ is the position of the knee and $\lambda_{K}$ is lower leg length, as $\mathbf{u}_{K}(t)=\left[-\sin \left(\theta_{T}(t)-\theta_{K}(t)\right), \cos \left(\theta_{T}(t)-\theta_{K}(t)\right)\right]$ and $\mathbf{k}(t)=\left[k_{x}\right.$, $k_{y}$, where $k_{x}$ and $k_{y}$ is the point of the knee. Decomposing Eq. (7) into $x$ and $y$ parts yields the coordinates of the ankle as,

$$
\begin{aligned}
& a_{x}(t)=k_{x}(t)-\lambda_{K} \sin \left(\theta_{T}(t)-\theta_{K}(t)\right) \\
& a_{y}(t)=k_{y}(t)+\lambda_{K} \cos \left(\theta_{T}(t)-\theta_{K}(t)\right)
\end{aligned}
$$

\subsection{Feature Extraction, Fourier Description and $k$-Nearest Neighbour}

Equations (5, 6, 8 and 9), which describe the model of the moving leg, are used as the basis for feature extraction. The parameters of interest are $h_{x}(0), h_{y}(0), A_{T}, A_{K 1}, A_{K 2}$, $A_{y}, C_{T}, C_{K 1}, C_{K 2}, \lambda_{T}, \lambda_{K}$ and $\phi$. With this model, the computational cost and the number of parameters required is greatly reduced as compared with the earlier modelbased approach $^{[8]}$, which requires at least 26 parameters to approximate the motion of a single leg, not to mention both legs. With appropriate phase-lock, the model can handle both the left and right legs with the same number of parameters. By incorporating the coupled oscillators, the moving legs can be extracted accurately without confusion. Template matching is used frame by frame to determine the best values for the parameters and hence the angle of the line that best matches the edge data. These angles are then used to compute a Fourier transform representing the spectrum of variation in the thigh and lower leg.

The first and the second harmonic of the Fourier components of both thigh and knee rotations have the highest inter-class variance as compared with the higher components, which drop to near zero as the cut-off frequency of human walking is 5Hz. Multiplying the magnitude and phase component, i.e. phase-weighted magnitude, increases the inter-class variance. Hence, the first and second phaseweighted magnitude of both thigh and knee are used to create the gait signature. A basic classifier, the $k$-nearest neighbour was used. Clearly, other classifiers can be used to improve recognition capability but the issues here are rather more basic in nature. The Euclidean distance is used to obtain the distance between the test sample and the training data. The recognition results were evaluated by leave-one-out cross validation for different values of $k$ in the $k$-nearest neighbour rule. 


\section{Results}

The database consists of the side views of 5 subjects, each with 5 sequences of running and of walking on an electric treadmill with speeds set at 3 and $6 \mathrm{mph}$. The subjects wore their own choice of clothing. The inclination of the thigh and knee, as extracted by the new automatic coupled oscillator technique, were compared with manually labelled angles of the grey level images. This analysis showed that the average difference of the automatically labelled data was $2.5^{\circ}$. This is reflected in Fig. 3 , which shows the extracted position of the front of the leg superimposed on the original images. The extracted angles are still precise in regions where the legs cross and occlude each other and tolerates well with the nature of the background, especially occlusion by the treadmill's support.

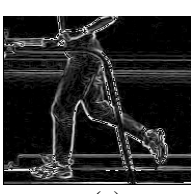

(a)

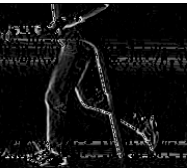

(b)

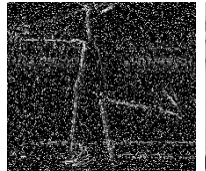

(c)

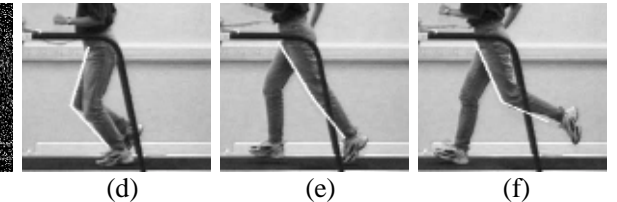

Fig. 3. (a) Edge data, (b) only leading edge is taken, (c) edge with $25 \%$ noise and (d-f) feature extraction results (superimposed in white).

Fig. 4 shows the phase-weighted magnitude of the Fourier component obtained from both the thigh and knee rotation. These are just three out of the four components used for recognition. The descriptors of walking subjects appear to cluster well, and are consistent with high recognition rates. The clusters for the running subjects are less distinct, though a more sophisticated classifier could handle such data, especially when complemented by other descriptors.
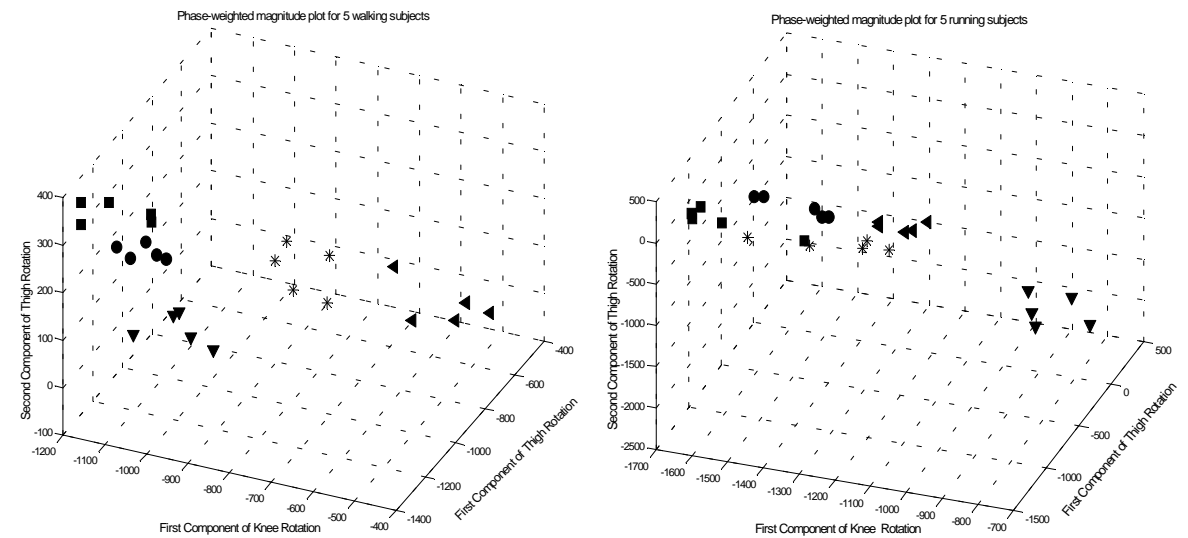

Fig. 4 (a) and (b) shows the phase-weighted magnitude obtained from the Fourier transform of 5 walking and running subjects, respectively.

The recognition rates are very encouraging and reach $96 \%$ for walking and $92 \%$ for running. These are consistent with other studies ${ }^{[5,7]}$ on similarly sized databases. The effect of smoothing in the feature space by using larger values of $k$ is only seen in 
the noisy running data. In other cases the nature of the feature space clusters lead to limited effect. On this data, it appears that $k=3$ is the most prudent choice in general. With $50 \%$ added noise, the rate maintains at an acceptable level, which reaches $80 \%$ and $76 \%$ for walking and running respectively. Table 1 shows the classification rates.

\begin{tabular}{|c|c|c|c|c|c|c|}
\hline Noise Level (\%) & \multicolumn{3}{|c|}{ Walking (\%) } & \multicolumn{3}{c|}{ Running (\%) } \\
\hline & $\boldsymbol{k}=\mathbf{1}$ & $\boldsymbol{k = 3}$ & $\boldsymbol{k = 5}$ & $\boldsymbol{k = 1}$ & $\boldsymbol{k = 3}$ & $\boldsymbol{k = 5}$ \\
\hline 0 & 96 & 96 & 96 & 92 & 88 & 84 \\
\hline 25 & 80 & 88 & 88 & 84 & 84 & 88 \\
\hline 50 & 80 & 80 & 68 & 64 & 72 & 76 \\
\hline
\end{tabular}

Table 1. The classification rates via $k$-nearest neighbour for walking and running, with noise level of $0 \%, 25 \%$ and $50 \%$, with $k=1, k=3$ and $k=5$.

\section{Conclusions}

A new model based technique has been developed for the thigh and lower leg and achieves fewer parameters by using the property of coupled oscillators. This new model has been shown to good effect in recognition of subjects by the way they walk and by the way they run, with a relatively better recognition rate for walking as compared to running. However, the recognition rate could be improved by using a more sophisticated classifier. Accordingly there is a potential for determining an invariance relationship between walking and running which in turn could be used to recognise people by either the way they walk or run.

\section{References}

1. M. P. Murray, A. B. Drought and R. C. Kory. Walking Pattern of Normal Men. J. Bone and Joint Surgery, 46-A(2), 335-360, 1964.

2. L. Bianchi, D. Angeloni and F. Lacquaniti, Individual characteristics of human walking mechanics, Pflügers Arch - Eur J Physiol, 436, 343 - 356, 1998.

3. S.A. Niyogi and E.H. Adelson. Analyzing and Recognizing Walking Figures in XYT. Proc. IEEE Comp. Soc. Conf. on Comp. Vision and Patt. Recog., 469-474, 1994.

4. H. Murase and R. Sakai. Moving Object Recognition in Eigenspace Representation: Gait Analysis and Lip Reading. Patt. Recog. Letters, 17, 156-162, 1996.

5. J. Little and J. Boyd, Recognizing People by Their Gait: The Shape of Motion. MIT Press Journal-Videre, 1(2), 1-32, 1998.

6. P. S. Huang, C. J. Harris and M. S. Nixon. Human gait recognition in canonical space using temporal templates. IEE Proc.: VISP, 146(2), 93-100, 1999.

7. D. Cunado, M. S. Nixon and J. N. Carter. Automatic Gait Recognition via ModelBased Evidence Gathering. AutoID'99 Proc.: Workshop on Auto. Identification Adv. Tech., 27-30, October 1999.

8. L. Li, E. C. H. van den Bogert, G. E. Caldwell, R. E. A. van Emmerik, J. Hamill, Coordination Patterns of Walking and Running at Similar Speed and Stride Frequency, Human Mov. Sc., 18, 67-85, 1999.

9. I. Stewart. Symmetry-breaking cascades and the dynamics of morphogenesis and behaviour. Sc. Progress, 82(1), 9-48,1999. 